\title{
Isolation and Variation in Virulence of Single-Spore Isolates of Plasmodiophora brassicae from Canada
}

\author{
S. Xue and T. Cao, Department of Agricultural, Food and Nutritional Science, University of Alberta, Edmonton, AB \\ T6G 2P5, Canada; R. J. Howard, Alberta Agriculture and Food, Crop Diversification Centre South, Brooks, AB, \\ Canada; S. F. Hwang, Alberta Agriculture and Food, Crop Diversification Centre North, Edmonton, AB, Canada; \\ and S. E. Strelkov, Department of Agricultural, Food and Nutritional Science, University of Alberta, Edmonton
}

\begin{abstract}
Xue, S., Cao, T., Howard, R. J., Hwang, S. F., and Strelkov, S. E. 2008. Isolation and variation in virulence of single-spore isolates of Plasmodiophora brassicae from Canada. Plant Dis. 92:456462 .

Clubroot of crucifers, caused by Plasmodiophora brassicae, is emerging as an important disease of canola (Brassica napus) in Alberta, Canada. Populations of the pathogen often consist of a mixture of different pathotypes. Therefore, a simple and efficient method to isolate single resting spores of $P$. brassicae was developed, based on serial dilution of spore suspensions. The virulence of 24 single-spore isolates, representing five populations of the pathogen from Alberta, Ontario, and British Columbia, was characterized on the differentials of Williams and Somé et al. Symptoms were rated 6 weeks after inoculation and Fisher's least significant difference $(P<$ $0.05)$ was used to differentiate resistant from susceptible host reactions. The pathotype composition of $P$. brassicae in Canada appeared more diverse when single-spore isolates were examined rather than populations of the pathogen. In Alberta, at least three and possibly four pathotypes were identified among the 14 isolates tested, whereas a maximum of only two pathotypes had been reported previously when populations of the pathogen were examined. Pathotype 3 or $\mathrm{P}_{2}$, as classified on the differentials of Williams and Somé et al., respectively, was found to be predominant in the province. The occurrence of other pathotypes at lower frequencies suggests that caution should be used in any breeding strategy, because rare pathotypes of $P$. brassicae may quickly become predominant if susceptible host genotypes are continuously grown.
\end{abstract}

Additional keywords: Chinese cabbage, oilseed rape, races

As a soilborne biotrophic pathogen, Plasmodiophora brassicae Woronin causes clubroot, a serious disease affecting cruciferous crops worldwide. In Canada, clubroot traditionally has been a major problem in cole crop production in certain areas of British Columbia, Quebec, Ontario, and the Maritime provinces (18). However, clubroot also was identified recently on canola (Brassica napus L.) in Alberta $(26,29)$ and Quebec (16). In Alberta, the disease was found initially in 2003, in 12 commercial fields near St. Albert and in an experimental field in northeast Edmonton. However, additional surveys in 2005 and 2006 revealed more than 110 infested canola fields, which are distributed over a fairly wide geographic area in central Alberta $(23,24)$. The rapid increase in the number of clubroot-infested fields has raised concerns, because canola is one of the major crops in the province, one-third of which traditionally is planted in acidic

Corresponding author: S. E. Strelkov

E-mail: stephen.strelkov@ualberta.ca

Accepted for publication 2 November 2007.

doi:10.1094/PDIS-92-3-0456

(C) 2008 The American Phytopathological Society soils that favor disease development (29). In a study from Quebec (16), it was found that losses were as high as $91 \%$ when canola was planted in $P$. brassicae-infested soil, with a 4.7 to $6.1 \%$ reduction in oil content in infected plants. In Alberta, a yield loss of $100 \%$ occurred in one infested field in 2006, which had been consecutively cropped to canola for 2 years (24).

Crop rotation is the simplest and most effective approach for the management of clubroot, but may not be practical due to the longevity of the pathogen in infested soil (11). Application of lime suppresses disease development but is not economically feasible because large quantities of lime and repeated applications are required (6). The effect of fungicide treatment is not always consistent (15) and may be prohibitively expensive. Although the application of calcium cyanamide is considered an effective management option (12), it is also expensive. Furthermore, its efficacy has not been confirmed in a canola cropping system under prairie conditions. To date, no clubroot-resistant canola cultivars are available in Canada $(16,27)$, although the development of such cultivars is a desirable alternative approach for controlling this disease (13).

A good breeding strategy and appropriate deployment of plant resistance require an understanding of the diversity in the virulence of $P$. brassicae (14). The pathogenic diversity in field populations of $P$. brassicae from North America has been evaluated by a number of researchers $(1,5,20,25,27,33)$. Among the various systems proposed for pathotype designations, the differentials of Williams (33) commonly have been used to characterize pathogen populations from Canada $(5,17,27,33)$. In Europe, the European Clubroot Differential (ECD) set, which consists of 15 genotypes from three Brassica spp., has been used frequently $(3,4,32)$. Somé et al. (22) proposed a differential set consisting of three $B$. napus genotypes which may be useful for characterizing the pathogen in Alberta, where it occurs predominantly on $B$. napus canola.

Previously, nine $P$. brassicae field populations from various regions of Canada were tested using two sets of differential hosts (27). Seven populations from Alberta were classified as pathotypes 3 and 5 based on their reactions on the differential hosts of Williams (33), or as ECD16/15/12 and ECD16/15/0, respectively, on the ECD set (3). Two other populations, originating from Abbottsford, BC, and Orton, ON, were classified as pathotype 6 . Concerns, however, were raised over the intermediate and fluctuating results of certain differential hosts following inoculation with the populations tested (27). This phenomenon, which also was reported by others $(13,31,33)$, may result from heterogeneity in the pathogen populations $(31,33)$ or from genetic heterogeneity in the differential hosts themselves (13). The use of single-spore-derived isolates of $P$. brassicae has been suggested as a way to more accurately assess virulence in the pathogen, and a number of agarose-based methods for the isolation of single resting spores have been developed $(9,22,30)$. However, these agarose-based techniques are generally time consuming (10), and inoculation efficiencies can be very low. Therefore, the objectives of the present study were to (i) develop a simple and efficient technique for isolating single spores of $P$. brassicae and (ii) assess variation in virulence among single-spore isolates derived from pathogen populations from Canada. 


\section{MATERIALS AND METHODS}

Terminology. A "population" of P. brassicae refers to a collection of resting spores recovered from infested soil or clubs of an infected plant and used to inoculate a set of differential hosts (3), whereas a "single-spore isolate" refers to a population derived from a club inoculated with a single resting spore and maintained in isolation (32). To conform with the suggestions of Voorips (32) and Crute et al. (4), the term "pathotype" will be used instead of "race," regardless of the authors' original terminology.

Pathogen populations. Five populations of $P$. brassicae were used for the isolation of single resting spores of the pathogen: (i) SACAN03-1, originally obtained from clubbed canola roots collected near St. Albert, AB; (ii) CDCN04-1, collected from a diseased volunteer canola plant growing near the Crop Diversification Centre North, Alberta Agriculture and Food, Edmonton, AB; (iii) Leduc-1, recovered from infested soil from a market garden near Leduc, AB; (iv) ORCA04, collected from an infected cabbage root (Brassica oleracea L. var. capitata) from Orton, ON (provided by K. Callow, Ontario Ministry of Agriculture and Food, Guelph, ON); and (v) AbotJE04-1, recovered from infested soil collected near Abbottsford, BC (supplied by J. Elmhirst, Elmhirst Diagnostics, Abbotsford, BC). These populations, which previously were characterized by Strelkov et al. (27), were reproduced and maintained on the universally susceptible Chinese cabbage Granaat (B. rapa L. subsp. pekinensis).

Plant materials. The virulence of 25 single-spore isolates of $P$. brassicae was investigated following inoculation of six differential hosts. The differentials of Williams (33), purchased from the Crucifer Genetics Cooperative (Madison, WI), include the rutabaga (B. napus var. napobrassica Mill.) cvs. Wilhelmsburger and Laurentian and the cabbage cvs. Jersey Queen and Badger Shipper. The differentials of Somé et al. (22) include Wilhelmsburger and the oilseed rape cvs. Nevin and Brutor (B. napus L. var. napus). Nevin was obtained from the Czech Genebank, Research Institute of Crop Production, Prague-Ruzyne, Czech Republic, and Brutor from the Leibniz Institute of Plant Genetics and Crop Research (IPK) Genebank, Gatersleben, Germany. Chinese cabbage Granaat was purchased from B \& T World Seeds (Paguignan, France) and used as a susceptible control.

Isolation of single spores and host inoculation. Single spores were isolated from 8-week-old galls of Granaat inoculated with the individual populations of $P$. brassicae. Approximately $3 \mathrm{~g}$ of clubbed roots, either freshly harvested, frozen $\left(-80^{\circ} \mathrm{C}\right)$, or partially decayed (kept for about 2 months at $4^{\circ} \mathrm{C}$ in a sealed container), were homogenized in $50 \mathrm{ml}$ of sterile distilled water $\left(\mathrm{sdH}_{2} \mathrm{O}\right)$ in a commercial blender, followed by filtration through eight layers of cheesecloth (American Fiber \& Finishing Inc., Albemarle, NC). A pellet of resting spores was recovered from the filtrate by centrifugation $\left(1,000 \times g, 4^{\circ} \mathrm{C}, 10 \mathrm{~min}\right)$ and washed five times with $\mathrm{sdH}_{2} \mathrm{O}$, with the pellet resuspended and centrifuged each time. The final pellet was stored at $4^{\circ} \mathrm{C}$ for no more than 2 days before use.

Immediately prior to use, resting spores were resuspended in either sodium phosphate buffer $(\mathrm{pH} 8.0,10 \mathrm{mM})$ or in $5 \%$ ( vol/vol) glycerol in $\mathrm{sdH}_{2} \mathrm{O}$. The spore concentration was quantified using a hemacytometer (VWR, Mississauga, ON, Canada) and adjusted to approximately $2 \times$ $10^{3}$ spores $/ \mathrm{ml}$ with phosphate buffer or $5 \%$ ( $\mathrm{vol} / \mathrm{vol}$ ) glycerol. A $0.5-\mu \mathrm{l}$ drop of spore suspension was placed on the glass coverslip of a microscope slide (Fisher Scientific, Edmonton, AB) and examined at 100fold magnification. Upon confirmation of the presence of a single spore, the droplet was soaked up by gently moving the roots of a 1- or 2-week-old Granaat seedling (pre-germinated on moistened sterile filter paper in a petri dish) horizontally through the drop. The seedling then was placed in a petri dish on a piece of filter paper moistened with $\mathrm{sdH}_{2} \mathrm{O}$ (pH 6.5 to 7.0) or tap water $(\mathrm{pH} 6.0)$, or in a petri dish with the roots soaked in $100 \mu$ of $\mathrm{sdH}_{2} \mathrm{O}(\mathrm{pH} 6.5$ to 7.0) or tap water ( $\mathrm{pH} 6.0)$. The $\mathrm{pH}$ of the tap water was adjusted using $\mathrm{HCl}$. Following a 2-day incubation in darkness at $21^{\circ} \mathrm{C}$ (the incubation solution was replenished on the second day), the seedlings were transplanted into 7.5-cm-diameter plastic pots filled with Metro-Mix 290 soil (Scotts, Columbus, OH), at a density of one plant per pot, and maintained in a growth cabinet at 24 and $18^{\circ} \mathrm{C}$ (in light and darkness, respectively) with a 16-h photoperiod and a light intensity of 180 $\mu \mathrm{mol} / \mathrm{m}^{2} / \mathrm{s}$. The soil was kept saturated for 19 days after transplanting by soaking the pots in a layer of low-pH (6.0) tap water. The plants were watered thereafter as required and fertilized (15N-30P-15K) once a week.

Inoculation of differential hosts. Resting spores were collected (as described above) from the galled roots of 8-week-old Granaat plants inoculated with singlespore isolates of $P$. brassicae, and the spore concentration was diluted to approximately $1 \times 10^{7}$ spores $/ \mathrm{ml}$ with $\mathrm{sdH}_{2} \mathrm{O}$. One-week-old seedlings, which were pregerminated on moistened sterile filter paper, were inoculated by dipping the roots in the spore suspension for $10 \mathrm{~s} \mathrm{(27).} \mathrm{The}$ inoculated seedlings then were planted immediately in 4-by-4-cm plastic pots filled with Metro-Mix 290 soil, placed in flat containers without drainage holes at the bottom, and transferred to a greenhouse maintained at 21 and $18^{\circ} \mathrm{C}$ (in light and darkness, respectively) with a 16-h photoperiod (natural light supplemented by high-pressure sodium light). The soil was kept saturated using tap water $(\mathrm{pH} \mathrm{6.0)} \mathrm{for}$ the first week after inoculation, and then watered with regular tap water as required. Fertilizer (15N-30P-15K) was applied once a week.

Disease assessment. Granaat plants were assessed for symptom development 8 weeks after inoculation with single spores while the differential hosts were examined 6 weeks after inoculation for pathotype designation. The plants were pulled from the soil and then the roots were washed free of soil mix and visually evaluated for disease development using a 0-to-3 scale (13), where $0=$ no galling, $1=$ a few small galls (small galls on less than one-third of the roots), 2 = moderate galling (small to medium-sized galls on one-third to twothirds of the roots), and $3=$ severe galling (medium to large-sized galls on more than two-thirds of the roots). An index of disease (ID) was calculated using the formula of Horiuchi and Hori (8) as modified by Strelkov et al. (27):

$I D(\%)=\frac{\sum(n \times 0+n \times 1+n \times 2+n \times 3)}{N \times 3} \times 100 \%$

where $n$ is the number of plants in a class; $N$ is the total number of plants in an experimental unit; and $0,1,2$, and 3 are the symptom severity classes. The mass of the clubbed roots (total root mass minus mass of nonaffected roots) also was recorded for each infected plant.

Experimental design and data analysis. For each combination of inoculation method and population, 20 to 118 Granaat seedlings were inoculated with a suspension containing a single resting spore of $P$. brassicae. The number of seedlings inoculated and the number of galled plants obtained were recorded upon completion of the 8-week incubation period. The proportion of infected plants first was analyzed for the various populations and inoculation methods (Table 1) using the PROC RELIABILITY in SAS software (version 9.1; SAS Institute Inc., Cary, NC), to test the hypothesis of equality of the proportions (i.e., that the proportion of infected plants was not significantly different). For any given rejection of the hypothesis, the PROC MULTTEST then was used to contrast the infection rates if there were more than two proportions.

For characterization of the virulence of the single-spore-derived isolates on the differential hosts, five separate tests were conducted, each involving five different single-spore isolates used separately to inoculate the six differential hosts. For each combination of host and single-spore isolate, experimental units consisted of 12 plants and were replicated three times in a split-plot design with isolates as main plots and differential hosts as subplots. Because normal score plotting indicated that $90 \%$ of the data points (ID values) were nor- 
mally distributed, analysis of variance (ANOVA) was conducted, and Fisher's least significant difference (LSD) at a significance of $P<0.05$ was calculated to differentiate resistant from susceptible reactions. Genotypes developing IDs that were not significantly different from the genotype with the lowest ID in the same treatment were regarded as resistant, whereas those with IDs that were significantly different from the genotype with the lowest ID were regarded as susceptible. In addition, nonparametric analysis was conducted by transforming the mean disease ratings of each experimental unit into midranks (21), followed by analysis using the PROC MIXED in SAS software (version 9.1). Finally, the mass of clubbed roots ( 6 weeks after inoculation with the singlespore isolates) also was analyzed parametrically for each host genotype using the PROC MIXED.

\section{RESULTS}

Single-spore isolation. The infection rates obtained with the different populations and inoculation methods (Table 1) were treated as proportions and the PROC RELIABILITY was used to test the hypothesis of equality of the proportions. The proportion of infected plants was found to be significantly different across the five populations $\left(\chi^{2}=11.598, P<0.0206\right)$, and further analysis using the PROC MULTTEST revealed that the only significant contrast $(P<0.0216)$ for overall infection rate was between SACAN03-1 (8.4\%) and Leduc-1 (1.9\%). The hypothesis of equality of proportions also was rejected within the populations SACAN03-1 $\left(\chi^{2}=\right.$ 10.5685, $P<0.0051)$, AbotJE04-1 $\left(\chi^{2}=\right.$
4.9708, $P<0.0258)$, and ORCA04 $\left(\chi^{2}=\right.$ 18.6723, $P<0.0001)$, indicating that the infection rates obtained within these populations were significantly different. In contrast, testing of the hypothesis of equality revealed no significance (and therefore acceptance of the hypothesis) within the populations Leduc-1 $\left(\chi^{2}=3.2126, P>\right.$ $0.2006)$ and $\mathrm{CDCN} 04-1\left(\chi^{2}=6.3911, P>\right.$ 0.1718 ).

When the data were pooled across populations according to the type of water used for incubation of the seedlings, a significant difference $\left(\chi^{2}=13.4568, P<0.0002\right)$ was found between low-pH and distilled water. Infection rates of 9.0 and $2.8 \%$ were obtained with the distilled and low-pH water, respectively. However, within the population SACAN03-1, the type of water did not have a significant effect $\left(\chi^{2}=\right.$ $0.6836, P>0.4083)$. When the infection rates were pooled across populations according to buffer $\left(\chi^{2}=2.1314, P>\right.$ $0.1443)$, seedling age $\left(\chi^{2}=0.9029, P>\right.$ 0.3420 ), or the condition of the galls used for single-spore isolation $\left(\chi^{2}=2.6793, P>\right.$ 0.2619 ), no significant differences were found. Nevertheless, differences with respect to these parameters were observed within some populations. Within population SACAN03-1, the infection rate was significantly higher $\left(\chi^{2}=6.9446, P<\right.$ 0.0084 ) when using 2 -week-old seedlings $(13.25 \%)$ instead of 1-week-old seedlings $(0 \%)$. A significant difference $\left(\chi^{2}=\right.$ 18.6723, $P<0.0001)$ also was observed within ORCA04 when decomposing rather than fresh or frozen galls were used for isolation of resting spores. In the case of inoculations conducted with single spores derived from AbotJE04-1, the use of fresh rather than frozen galls significantly $\left(\chi^{2}=\right.$ 4.9708, $P<0.0258)$ increased the infection rate (Table 1).

Characterization of single-spore isolates. The virulence of five single-spore isolates derived from each of the five $P$. brassicae populations was compared on the differential hosts of Williams (33) and Somé et al. (22). ANOVA revealed no significant interactions between the differential hosts and the single-spore isolates derived from SACAN03-1 (SACAN-ss1 to SACAN-ss5) $(P>0.8458)$ or any significant interactions between the differential hosts and the single-spore isolates from AbotJE04-1 (AbotJE-ss1 to AbotJE-ss5; $P$ $>0.9999)$. Therefore, the five single-spore isolates derived from each of the two populations appeared to be fairly homogeneous (Table 2). The reactions of the hosts were classified as resistant or susceptible through LSD grouping $(P<0.05)$, with any genotype in the same group as the genotype with the lowest ID considered resistant. Using this criterion, two hosts, Wilhelmsburger and Badger Shipper, were classified as resistant to isolates SACANss1, SACAN-ss2, SACAN-ss4, and SACAN-ss5, corresponding to a pathotype 3 or $\mathrm{P}_{2}$ designation on the differentials of Williams (33) or Somé et al. (22), respectively (Table 3). However, only Wilhelmsburger was classified as resistant to SACAN-ss3, corresponding to a pathotype 2 designation on the hosts of Williams (33), but which would still correlate to $\mathrm{P}_{2}$ on the system of Somé et al. (22). Thus, although ANOVA indicated a nonsignificant interaction between differential hosts and isolates derived from SACAN03-1, the LSD groupings suggested some diversity. In

Table 1. Infection rates obtained for 1- and 2-week-old seedlings of Chinese cabbage cv. Granaat inoculated with single spores of Plasmodiophora brassicae under different conditions

\begin{tabular}{|c|c|c|c|c|c|}
\hline Population $^{v}$ & $\begin{array}{l}\text { Seedling age } \\
\text { (weeks) }\end{array}$ & $\begin{array}{l}\text { Clubs used for } \\
\text { isolation }^{w}\end{array}$ & Spore suspension $^{x}$ & $\begin{array}{l}\text { Water for } \\
\text { incubation }\end{array}$ & $\begin{array}{c}\text { Infection rate }(\%) \\
\text { (no. infected/total inoculated) }{ }^{\mathrm{z}}\end{array}$ \\
\hline \multirow[t]{3}{*}{ SACAN03-1 } & 1 & Frozen & Phosphate buffer & Low $\mathrm{pH}$ & $0.00(0 / 48) \mathrm{a}$ \\
\hline & 2 & Frozen & Phosphate buffer & Distilled & $4.17(1 / 24) \mathrm{ab}$ \\
\hline & 2 & Frozen & Phosphate buffer & Low pH & $16.95(10 / 59) \mathrm{b}$ \\
\hline \multirow[t]{2}{*}{ AbotJE04-1 } & 2 & Frozen & Phosphate buffer & Low $\mathrm{pH}$ & $0.00(0 / 77) \mathrm{a}$ \\
\hline & 2 & Fresh & Phosphate buffer & Low $\mathrm{pH}$ & $6.25(5 / 80) b$ \\
\hline \multirow[t]{3}{*}{ Leduc-1 } & 2 & Frozen & Phosphate buffer & Low $\mathrm{pH}$ & $0.00(0 / 75) \mathrm{a}$ \\
\hline & 2 & Fresh & Phosphate buffer & Low pH & $1.25(1 / 80) \mathrm{a}$ \\
\hline & 2 & Decomposing & Phosphate buffer & Low pH & $3.45(4 / 116) \mathrm{a}$ \\
\hline \multirow[t]{3}{*}{ ORCA04 } & 2 & Frozen & Phosphate buffer & Low pH & $0.00(0 / 118) \mathrm{a}$ \\
\hline & 2 & Fresh & Phosphate buffer & Low $\mathrm{pH}$ & $0.00(0 / 63) \mathrm{a}$ \\
\hline & 2 & Decomposing & Phosphate buffer & Distilled & $10.00(8 / 80) b$ \\
\hline \multirow{5}{*}{ CDCN04-1 } & 2 & Decomposing & Phosphate buffer & Low $\mathrm{pH}$ & $0.92(1 / 109) \mathrm{a}$ \\
\hline & 2 & Fresh & Phosphate buffer & Low pH & $4.00(2 / 50) \mathrm{a}$ \\
\hline & 1 & Decomposing & $5 \%$ glycerol & Low pH & $5.00(1 / 20) \mathrm{a}$ \\
\hline & 2 & Decomposing & $5 \%$ glycerol & Low $\mathrm{pH}$ & $4.08(2 / 49) \mathrm{a}$ \\
\hline & 2 & Decomposing & $5 \%$ glycerol & Distilled & $10.34(3 / 29) \mathrm{a}$ \\
\hline
\end{tabular}

v Populations were maintained on Chinese cabbage cv. Granaat.

${ }^{\text {w }}$ Frozen clubs were stored at $-80^{\circ} \mathrm{C}$, fresh clubs were used 1 or 2 days after harvest (stored at $4^{\circ} \mathrm{C}$ prior to use), and decomposing clubs were stored 1 to 2 months at $4^{\circ} \mathrm{C}$ in parafilm-sealed beakers prior to use.

${ }^{x}$ Refers to solutions used to resuspend the resting spores prior to microscopic examination and inoculation of seedlings; phosphate buffer = sodium phosphate buffer $(\mathrm{pH} 8,10 \mathrm{mM}) ; 5 \%$ glycerol = 5\% ( $\mathrm{vol} / \mathrm{vol})$ glycerol in sterile distilled $\mathrm{H}_{2} \mathrm{O}$; both solutions were autoclaved prior to use.

${ }^{y}$ Refers to water used to incubate seedlings in petri dishes for 2 days after inoculation; low $\mathrm{pH}=$ tap water with $\mathrm{pH}$ adjusted to 6.0 ; distilled $=$ distilled water $(\mathrm{pH}=6.5$ to 7.0$)$; both low $\mathrm{pH}$ and distilled water were autoclaved prior to use.

${ }^{\mathrm{z}}$ Percentages followed by the same letter are not significantly different at $P<0.05$ within each population, as determined using the PROC RELIABILITY or PROC MULTTEST in SAS software (version 9.1; SAS Institute Inc., Cary, NC). 
response to inoculation with single-spore isolates derived from the population AbotJE04-1 (AbotJE-ss1 to AbotJE-ss5), only Brutor and Jersey Queen appeared susceptible. Therefore, isolates AbotJE-ss1 to AbotJE-ss5 were classified as pathotype 6 according to Williams (33) or as $\mathrm{P}_{3}$ on the differentials of Somé et al. (22).

Significant interactions were found between differential hosts and single-spore isolates derived from the populations CDCN04-1 $(P<0.0005)$, Leduc-1 $(P<$ $0.0001)$, and ORCA04 $(P<0.0001)$. Three of the five single-spore isolates from CDCN04-1 (CDCN-ss2, CDCN-ss4, and CDCN-ss5) possessed similar virulence patterns, with only Wilhemsburger and Badger Shipper showing resistance. Thus, these isolates were classified as pathotype 3 on the differentials of Williams (33) or as $\mathrm{P}_{2}$ on the hosts of Somé et al. (22) (Table $3)$. In contrast, one of the isolates, CDCNss3, exhibited reduced virulence on Jersey Queen in addition to its avirulence on Wilhemsburger and Badger Shipper (Table 2); this resulted in a pathotype 8 classification on the differentials of Williams (33) but still corresponded to a $\mathrm{P}_{2}$ designation on the system of Somé et al. (22) (Table 3). Similarly, isolate CDCN-ss1 also exhibited reduced virulence on Jersey Queen, resulting in a pathotype 8 designation according to Williams (33). However, the host differential Brutor also was resistant to $\mathrm{CDCN}$ ss1, producing a virulence pattern that was not described by Somé et al. (22) in their original report. Nonetheless, ManzanaresDauleux et al. (14) (using the differentials of Somé et al.; 22) identified isolates with this virulence pattern and classified them as a novel pathotype, $\mathrm{P}_{6}$, a designation that we have retained (Table 3 ).

The Leduc-1 population also appeared to be heterogeneous in its composition (Table 3). The single-spore isolates Leducss1, Leduc-ss4, and Leduc-ss5 were classified as pathotype 3 or $\mathrm{P}_{2}$ according to Williams (33) or Somé et al. (22), respectively, based on the resistant reactions of Wilhemsburger and Badger Shipper (Tables 2 and 3). In contrast, only Jersey Queen and Brutor were susceptible to Leduc-ss2, corresponding to a pathotype 6 designation on the system of Williams (33) or a $\mathrm{P}_{3}$ designation according to Somé et al. (22). Inoculation of the differential hosts with Leduc-ss 3 revealed that the viability of this isolate was poor, because even the universal suscept Granaat developed an ID of only $44 \%$ (Table 2). Hence, this isolate was excluded from further analysis. The hosts Wilhemsburger and Badger Shipper were resistant to the single-spore isolates ORCA-ss 1 and ORCA-ss5, derived from the population ORCA04. All other hosts were susceptible to these isolates, corresponding to a pathotype 3 designation according to Williams (33) or a $\mathrm{P}_{2}$ designation according to Somé et al. (22). Isolate ORCA-ss2, derived from the same popula- tion, was avirulent on Jersey Queen as well as on Wilhemsburger and Badger Shipper, which resulted in a pathotype 8 classification on the system of Williams (33). However, because Jersey Queen was not used as a differential by Somé et al. (22), ORCA-ss2 was still classified as $\mathrm{P}_{2}$ according to their system. The remaining single-spore isolates, ORCA-ss 3 and ORCA-ss4, were avirulent on all hosts except for Brutor, corresponding to a pathotype 5 or $\mathrm{P}_{3}$ designation on the differentials of Williams (33) and Somé et al. (22), respectively.

Parametric analysis of the average mass of clubbed roots in each experimental unit using the PROC MIXED of SAS yielded results very similar to those from the analysis of IDs, when pairwise comparisons of differential host within isolates were conducted using the most resistant host as a control. In contrast, the results obtained using nonparametric analysis of disease indices showed greater disparity with those from the analysis of clubbed root masses (data not shown). The highly significant positive correlation between IDs and masses of clubbed roots $\left(R^{2}=\right.$ 0.6746, $P<0.0001, N=5,152)$ also confirmed that the IDs accurately reflected the degree of disease development.

\section{DISCUSSION}

Single-spore isolation. Two technical problems, the minute size of $P$. brassicae

Table 2. Disease indices on six Brassica differential hosts following inoculation with single-spore-derived isolates of Plasmodiophora brassicae from Canada $^{\mathrm{x}}$

\begin{tabular}{|c|c|c|c|c|c|c|c|c|}
\hline \multirow[b]{2}{*}{ Single-spore isolate } & \multirow[b]{2}{*}{ Control (Granaat) } & \multicolumn{4}{|c|}{ Brassica napus cvs. } & \multicolumn{2}{|c|}{ B. oleracea cvs. } & \multirow[b]{2}{*}{$\mathbf{L S D}_{\mathbf{0 . 0 5}}$} \\
\hline & & Brutor & Laurentian & Nevin & Wilhelmsburger & Badger Shipper & Jersey Queen & \\
\hline SACAN-ss1 & 100 & 99.0 & 81.9 & 65.2 & $4.7 \mathrm{R}$ & $17.5 \mathrm{R}$ & 41.8 & 12.9 \\
\hline SACAN-ss 2 & 90.9 & 96.8 & 86.0 & 62.0 & $12.0 \mathrm{R}$ & $23.9 \mathrm{R}$ & 48.3 & 22.0 \\
\hline SACAN-ss $3^{y}$ & 97.2 & 96.7 & 67.0 & 62.5 & $8.0 \mathrm{R}$ & 31.6 & 44.0 & 20.9 \\
\hline SACAN-ss4 & 100 & 96.0 & 80.7 & 69.3 & $9.3 \mathrm{R}$ & $25.8 \mathrm{R}$ & 46.4 & 17.1 \\
\hline SACAN-ss5 & 100 & 97.7 & 82.0 & 67.7 & $17.8 \mathrm{R}$ & $24.3 \mathrm{R}$ & 39.3 & 16.6 \\
\hline AbotJE-ss 1 & 84.8 & 57.0 & $0.0 \mathrm{R}$ & $3.8 \mathrm{R}$ & $0.0 \mathrm{R}$ & $4.8 \mathrm{R}$ & 77.0 & 15.0 \\
\hline AbotJE-ss2 & 83.3 & 65.3 & $0.0 \mathrm{R}$ & $0.0 \mathrm{R}$ & $6.0 \mathrm{R}$ & $8.9 \mathrm{R}$ & 82.3 & 18.3 \\
\hline AbotJE-ss3 & 94.4 & 64.1 & $0.0 \mathrm{R}$ & $0.0 \mathrm{R}$ & $1.0 \mathrm{R}$ & $3.9 \mathrm{R}$ & 84.3 & 17.7 \\
\hline AbotJE-ss4 & 88.9 & 63.7 & $0.0 \mathrm{R}$ & $0.9 \mathrm{R}$ & $0.0 \mathrm{R}$ & $8.3 \mathrm{R}$ & 84.7 & 20.7 \\
\hline AbotJE-ss 5 & 88.9 & 62.8 & $0.0 \mathrm{R}$ & $1.9 \mathrm{R}$ & $0.0 \mathrm{R}$ & $5.5 \mathrm{R}$ & 85.7 & 12.0 \\
\hline CDCN-ss 1 & 72.2 & $17.7 \mathrm{R}$ & 37.8 & 68.5 & $8.3 \mathrm{R}$ & $10.2 \mathrm{R}$ & $21.4 \mathrm{R}$ & 29.2 \\
\hline CDCN-ss2 & 100 & 99.0 & 77.0 & 83.7 & $13.9 \mathrm{R}$ & $25.7 \mathrm{R}$ & 50.1 & 14.4 \\
\hline CDCN-ss 3 & 100 & 100 & 61.7 & 67.5 & $13.4 \mathrm{R}$ & $15.8 \mathrm{R}$ & $49.5 \mathrm{R}$ & 40.0 \\
\hline CDCN-ss 4 & 100 & 99.1 & 95.5 & 90.1 & $28.0 \mathrm{R}$ & $17.0 \mathrm{R}$ & 75.2 & 15.6 \\
\hline CDCN-ss5 & 100 & 97.8 & 95.1 & 85.3 & $19.5 \mathrm{R}$ & $6.1 \mathrm{R}$ & 48.6 & 24.0 \\
\hline Leduc-ss1 & 100 & 99.0 & 68.0 & 82.7 & 7.4R & $17.3 \mathrm{R}$ & 56.0 & 29.4 \\
\hline Leduc-ss2 & 91.6 & 16.6 & $0.0 \mathrm{R}$ & $0.0 \mathrm{R}$ & $0.0 \mathrm{R}$ & $2.8 \mathrm{R}$ & 80.7 & 14.0 \\
\hline Leduc-ss $3^{z}$ & 44.4 & 4.6 & 0.9 & 0.0 & 0.0 & 0.0 & 3.0 & 7.4 \\
\hline Leduc-ss4 & 97.2 & 93.7 & 24.0 & 74.7 & $0.9 \mathrm{R}$ & $4.6 \mathrm{R}$ & 30.8 & 7.4 \\
\hline Leduc-ss 5 & 100 & 99.1 & 83.1 & 79.5 & $16.7 \mathrm{R}$ & $2.8 \mathrm{R}$ & 55.2 & 31.6 \\
\hline ORCA-ss 1 & 100 & 100 & 61.7 & 73.3 & $5.3 \mathrm{R}$ & $25.7 \mathrm{R}$ & 48.0 & 21.7 \\
\hline ORCA-ss 2 & 96.7 & 94.0 & 48.5 & 79.0 & $5.5 \mathrm{R}$ & $3.7 \mathrm{R}$ & $26.8 \mathrm{R}$ & 23.3 \\
\hline ORCA-ss 3 & 72.7 & 24.7 & $3.7 \mathrm{R}$ & 21.3R & $0.0 \mathrm{R}$ & $0.0 \mathrm{R}$ & $2.8 \mathrm{R}$ & 21.8 \\
\hline ORCA-ss 4 & 66.7 & 33.4 & $1.9 \mathrm{R}$ & $14.2 \mathrm{R}$ & $0.9 \mathrm{R}$ & $0.9 \mathrm{R}$ & $9.4 \mathrm{R}$ & 22.3 \\
\hline ORCA-ss5 & 100 & 98.1 & 100 & 93.3 & $21.9 \mathrm{R}$ & $13.5 \mathrm{R}$ & 71.5 & 16.7 \\
\hline
\end{tabular}

${ }^{\mathrm{x}} \mathrm{R}=$ resistant reaction. Least significant difference (LSD; $P<0.05$ ) was used to differentiate resistant from susceptible reactions; genotypes developing disease indices (IDs) that were not significantly different from the genotype with the lowest ID in the same treatment were regarded as resistant, and all others were regarded as susceptible.

y Badger Shipper was only tentatively classified as susceptible to SACAN-ss3, because analysis of variance indicated no significant interactions between the differential hosts and SACAN-ss1 to SACAN-ss5.

z The reactions of the differential hosts to Leduc-ss3 were not classified, because the ID on the susceptible control (Chinese cabbage cv. Granaat) was only $44 \%$. 
resting spores and their tendency to aggregate, can hamper the isolation of single spores of the pathogen. These problems often have been dealt with by the use of agarose-based methods to isolate single spores of $P$. brassicae, which rely on the even distribution of resting spores on a thin film of agarose placed on a microscope slide (22); pieces of agarose containing a single resting spore are excised and used to inoculate host plants. To facilitate this process, special equipment, such as a dummy microscope objective lens on which a punch is mounted to cut and lift out a disc of agar containing a single spore, occasionally has been employed $(9,30)$. In the current study, we experienced great difficulties in lifting up tiny pieces of agarose without the aid of special apparatus. Moreover, the agarose attached to a spore might prevent it from making direct contact with the root hairs of the host.

An alternative to agarose-based methods is the use of highly diluted $P$. brassicae spore suspensions, in which the presence of a single resting spore can be confirmed per unit volume. For instance, Buczacki (2) located single spores of the pathogen in droplets of a diluted spore suspension placed in wells of a glass cavity slide, and withdrew and expelled the droplets onto the roots of seedlings when the presence of a single spore was confirmed. Using this procedure, however, a considerable proportion of the spore suspension was left in the pipette tips and in the wells of the slide, resulting in reduced infection rates. Kageyama et al. (10) deposited a 0.5- $\mu$ l droplet of spore suspension on the glass cover slip of a microscope slide. The presence of a single resting spore in the droplet was confirmed by microscopic examination, and the cover slip carrying the single spore was placed on the surface of autoclaved soil in a container. A 1-day-old Chinese cabbage seedling was laid over the spore droplet and covered with a small amount of additional soil. Although we evaluated this method, we obtained very low infection rates ( 0 to $4.2 \%$ ), perhaps as a result of the attachment of resting spores to the glass cover slip.

Therefore, one of the objectives of the current study was to develop a relatively simple and efficient method to isolate single resting spores of $P$. brassicae that allows for the inoculation and infection of host roots with increased success. The procedure we developed has three advantages: (i) the tendency for resting spores to aggregate was reduced by using $5 \%$ $(\mathrm{vol} / \mathrm{vol})$ glycerol in $\mathrm{sdH}_{2} \mathrm{O}$ for the preparation of the spore suspension, thereby facilitating the identification and isolation of single spores; (ii) the protocol allowed direct contact of the resting spore with the root hairs of the host, resulting in higher infection rates; and (iii) fewer technical difficulties were encountered because pieces of agarose did not have to be cut and removed. Using this procedure with a properly diluted spore suspension, two to four seedlings could be inoculated per hour, and infection rates ranging from 4 to $17 \%$ were obtained with the various pathogen populations (Table 1). The relative simplicity and efficiency of this technique should facilitate research that requires the use of single-spore-derived isolates of $P$. brassicae, including breeding for clubroot resistance and analysis of the genetic diversity of field populations using molecular markers.

A germination stimulating factor from Brassica root exudates has been proposed to stimulate germination of pathogen resting spores (28); therefore, we hypothesized that infection rates would be higher in 2week-old versus 1-week-old seedlings because, presumably, they would release more root exudates. However, although the data obtained with SACAN03-1 appeared to support such a hypothesis, the data obtained with CDCN04-1 did not (Table 1). Hence, additional experiments are required to clarify this issue. The condition of the galls used for the isolation of resting spores also had an inconsistent effect in the different populations (Table 1). In the case of ORCA04, spores obtained from decomposing galls produced significantly higher infection rates than spores from fresh or frozen galls. Suzuki et al. (28) reported similar results and hypothesized that resting spores from decomposing galls possessed higher rates of germination, perhaps because of increased maturity. However, in Leduc-1, inoculum from decomposing galls did not yield significantly higher infection rates and, when the data were

Table 3. Reactions of six Brassica differential hosts in response to inoculation with single-spore-derived isolates of Plasmodiophora brassicae from Canada ${ }^{\mathrm{x}}$

\begin{tabular}{|c|c|c|c|c|c|c|c|}
\hline \multirow[b]{2}{*}{ Single-spore isolate } & \multicolumn{4}{|c|}{ Brassica napus cvs. } & \multicolumn{2}{|c|}{ B. oleracea cvs. } & \multirow[b]{2}{*}{ Pathotype $^{y}$} \\
\hline & Brutor & Laurentian & Nevin & $\begin{array}{l}\text { Wilhelmsburger } \\
\text { nof }\end{array}$ & Badger Shipper & Jersey Queen & \\
\hline SACAN-ss1 & + & + & + & - & - & + & $3 / \mathrm{P}_{2}$ \\
\hline SACAN-ss 2 & + & + & + & - & - & + & $3 / \mathrm{P}_{2}$ \\
\hline SACAN-ss3 & + & + & + & - & - & + & $2 / \mathrm{P}_{2}^{\mathrm{z}}$ \\
\hline SACAN-ss4 & + & + & + & - & - & + & $3 / \mathrm{P}_{2}$ \\
\hline SACAN-ss5 & + & + & + & - & - & + & $3 / \mathrm{P}_{2}$ \\
\hline AbotJE-ss1 & + & - & - & _- & _- & + & $6 / \mathrm{P}_{3}$ \\
\hline AbotJE-ss2 & + & - & - & - & - & + & $6 / \mathrm{P}_{3}$ \\
\hline AbotJE-ss 3 & + & - & - & - & - & + & $6 / \mathrm{P}_{3}$ \\
\hline AbotJE-ss4 & + & - & - & - & - & + & $6 / \mathrm{P}_{3}$ \\
\hline AbotJE-ss5 & + & - & - & - & - & + & $6 / \mathrm{P}_{3}$ \\
\hline CDCN-ss1 & - & + & + & - & - & - & $8 / \mathrm{P}_{6}$ \\
\hline CDCN-ss2 & + & + & + & - & - & + & $3 / \mathrm{P}_{2}$ \\
\hline CDCN-ss3 & + & + & + & - & - & - & $8 / \mathrm{P}_{2}$ \\
\hline CDCN-ss4 & + & + & + & - & - & + & $3 / \mathrm{P}_{2}$ \\
\hline CDCN-ss5 & + & + & + & - & - & + & $3 / \mathrm{P}_{2}$ \\
\hline Leduc-ss1 & + & + & + & - & - & + & $3 / \mathrm{P}_{2}$ \\
\hline Leduc-ss2 & + & - & - & - & - & + & $6 / \mathrm{P}_{3}$ \\
\hline Leduc-ss4 & + & + & + & - & - & + & $3 / \mathrm{P}_{2}$ \\
\hline Leduc-ss5 & + & + & + & - & - & + & $3 / \mathrm{P}_{2}$ \\
\hline ORCA-ss1 & + & + & + & - & - & + & $3 / \mathrm{P}_{2}$ \\
\hline ORCA-ss2 & + & + & + & - & - & - & $8 / \mathrm{P}_{2}$ \\
\hline ORCA-ss3 & + & - & - & - & - & - & $5 / \mathrm{P}_{3}$ \\
\hline ORCA-ss4 & + & - & - & - & - & - & $5 / \mathrm{P}_{3}$ \\
\hline ORCA-ss5 & + & + & + & - & - & + & $3 / \mathrm{P}_{2}$ \\
\hline
\end{tabular}

${ }^{\mathrm{x}}$ Susceptible (compatible) and resistant (incompatible) host reactions are indicated by + and - , respectively. Least significant difference $($ LSD; $P<0.05$ ) was used to differentiate resistant from susceptible reactions; genotypes developing disease indices (IDs) that were not significantly different from the genotype with the lowest ID in the same treatment were regarded as resistant, and all others were regarded as susceptible.

y As determined on the differential hosts of Williams (33) and Somé et al. (22).

${ }^{z}$ SACAN-ss 3 was classified as pathotype 2 on the differentials of Williams based on LSD groupings, although analysis of variance indicated no significant interactions between the differential hosts and SACAN-ss1 to SACAN-ss5. 
pooled across populations, no significant difference between gall types was observed.

Characterization of single-sporederived isolates. Various systems have been proposed for the classification of $P$. brassicae into pathotypes or races. A recent report used the hosts of Williams (33) and the ECD set (3) to analyze the virulence of pathogen populations from Canada (27). In the current study, we retained the differentials of Williams (33) to enable comparisons between the virulence of single-spore isolates (examined here) and the populations from which they were obtained (27). However, because most isolates examined were obtained originally from canola (B. napus), we also included the $B$. napus differential Brutor, which allowed us to obtain pathotype designations according to Somé et al. (22). The differentials of the ECD set (3), with the exception of Chinese cabbage Granaat (ECD 05), were excluded from the current study because of space limitations; Granaat was retained as a susceptible control.

Although physiologic specialization has long been known in the pathogen (7), many of the differential hosts that have been proposed to characterize $P$. brassicae develop intermediate ID values in response to inoculation $(13,27,31)$. Intermediate and fluctuating IDs have been attributed not only to the heterogeneity of $P$. brassicae populations $(9,19,30)$ but also to the heterogeneity of the differential hosts themselves (13). The results from the current study do suggest that genetic heterogeneity in some hosts contributes to the development of indistinct reactions (31), because intermediate IDs often were obtained after inoculation with single-spore isolates that should represent a single genotype of the pathogen (Table 2). This was particularly evident with the cabbage Jersey Queen, which commonly developed IDs ranging from 30 to $60 \%$. However, in the case of this and other cabbage cultivars, indistinct reactions may result not only from genetic heterogeneity in the host genotypes but also from possible quantitative additive effects that have been reported for clubroot resistance in this species (4).

The occurrence of indistinct host reactions makes it difficult to classify the pathogen into races or pathotypes, because a qualitative designation (resistant or susceptible) has to be applied to what, in many instances, appears to be a quantitative reaction (25). A number of strategies have been used to accommodate indistinct host responses. Most recently, Strelkov and coworkers $(25,27)$ regarded an ID of $50 \%$ as the cut-off between a resistant and a susceptible reaction. However, this represents a somewhat arbitrary criterion, and other workers have used different cut-offs (for example, Somé et al. [22] used 25\%). In the current study, ANOVA of the IDs was conducted and LSD $(P<0.05)$ used to differentiate resistant from susceptible reactions (Table 2). For comparison, we also performed parametric analysis using the average mass of clubbed roots and nonparametric analysis using the mean disease ratings. Although parametric analysis of the mass of clubbed roots yielded results similar to those from the analysis of IDs, the results were very different from those of the nonparametric analysis. Given that the mass of clubs provides a biological measure of susceptibility, this suggests that parametric analysis is more appropriate than nonparametric analysis for study of the Brassica-P. brassicae interaction.

Using LSD $(P<0.05)$ to distinguish resistant from susceptible reactions, we found that the pathotype designation of most isolates obtained from the populations SACAN03-1, Leduc-1, and AbotJE04-1 were similar to the populations from which they were derived. In the case of AbotJE04-1, all single-spore isolates possessed the same virulence pattern as this population and were classified as pathotype 6 according to the system of Williams (33) or as $\mathrm{P}_{3}$ on the differentials of Somé et al. (22), suggesting that this population is fairly homogenous (Table 3 ). Similarly, ANOVA revealed no significant interaction between differential hosts and isolates derived from SACAN03-1, which previously was classified as pathotype 3 (27) on the differentials of Williams (33). However, although four of five singlespore isolates from this population shared a pathotype 3 designation based on LSD groupings, isolate SACAN-ss3 was classified as pathotype 2 because of a susceptible reaction by Badger Shipper (Table 3). Nevertheless, the actual ID of Badger Shipper in response to SACAN-ss3 was only 6 to $14 \%$ higher than in response to the other isolates, and further testing may be required to confirm whether SACANss3 does, in fact, represent a different pathotype. On the differentials of Somé et al. (22), which do not include Badger Shipper, all isolates derived from SACAN03-1 were classified as $\mathrm{P}_{2}$ (Table 3). In the case of Leduc-1, a population previously classified as pathotype 3 (27), three of four single-spore isolates also shared the same classification (equivalent to $\mathrm{P}_{2}$ on the differentials of Somé et al.; 22). However, isolate Leduc-ss2 was designated as pathotype 6 or $\mathrm{P}_{3}$ on the hosts of Williams (33) and Somé et al. (22), respectively. The different virulence patterns in isolates derived from Leduc-1 were confirmed by ANOVA, which indicated a significant interaction between differential hosts and single-spore isolates, strongly suggesting that heterogeneity exists in this population.

The isolates obtained from the other two P. brassicae populations, CDCN04-1 and ORCA04, possessed very different virulence patterns relative to the original popu- lations and also appeared to be a mixture of pathotypes. Whereas CDCN04-1 previously was classified as pathotype 5 (27) on the differentials of Williams (33), three of the five single-spore isolates tested were classified as pathotype 3 in the present study, with the remaining isolates classified as pathotype 8 (Table 3). However, Strelkov et al. (27) cautioned that the pathotype 5 classification obtained in their study was based largely on the reactions of the $B$. oleracea hosts, which produced intermediate and fluctuating reactions against all populations; hence, it is possible that this classification may have simply reflected the inconsistent reactions of these hosts. Nonetheless, Laurentian, a B. napus host that generally produces distinct reactions, was more susceptible to the singlespore isolates than to the original population, and the discrepancy in the pathotype designations between CDCN04-1 and its derived single-spore isolates may have been due to the interactions of different pathotypes in the original population $(9,31)$. One pathotype can alter the response of a differential host to a second pathotype present in the same inoculum (9), and indistinct reactions may result from the presence of multiple pathotypes in the same population $(31,33)$. On the hosts of Somé et al. (22), four of the isolates from CDCN04-1 were classified as $\mathrm{P}_{2}$, while the fifth (CDCN-ss1) was classified as $\mathrm{P}_{6}$ (Table 3 ).

Similarly, whereas ORCA04-1 had been classified as pathotype 6 (27) on the hosts of Williams (33), none of the single-spore isolates from this population shared that designation (Table 3). Two of the five isolates were classified as pathotype 3 , two were classified as pathotype 5 , and one was classified as pathotype 8 . On the differentials of Somé et al. (22), three isolates were designated as $\mathrm{P}_{2}$ and two were classified as $\mathrm{P}_{3}$. The differential classification of three of the isolates (ORCA-ss1, ORCAss2, and ORCA-ss5) resulted in part from the fact that they exhibited greatly increased virulence on Nevin and Laurentian (Table 2) relative to the original population (27). The lower virulence of the latter may have been due to its heterogeneous composition, because reduced virulence has been reported previously in pathotype mixtures and may reflect competition between pathogenic and nonpathogenic isolates (32). Nonpathogenic isolates also may induce resistance mechanisms in the host, thereby reducing infection by pathogenic isolates. A reduction in virulence resulting from a pathotype mixture cannot explain the results obtained with ORCA-ss3 and ORCA-ss4, however, because these isolates (and ORCA-ss2) exhibited greatly reduced virulence on Jersey Queen relative to the original population (Table 2). It is possible that nonpathogenic isolates also can rely on pathogenic isolates present in the same population to proliferate within 
otherwise resistant hosts. Clearly, complex interactions between pathotypes and host genotypes appear to be at play.

The pathotype composition of $P$. brassicae in Canada appeared more diverse when single-spore isolates rather than populations of the pathogen were examined. In Alberta, at least three and possibly four pathotypes were identified among 14 isolates characterized on two differential sets; these isolates were derived from a small number of populations, and additional pathotypes may be detected with further testing. In contrast, only two pathotypes were found when populations of $P$. brassicae were tested (27), and another study found only one (25). Nevertheless, the predominant pathotype among isolates from Alberta remains pathotype 3 or $\mathrm{P}_{2}$, as classified on the differentials of Williams (33) and Somé et al. (22), respectively. This is consistent with studies of field populations, in which pathotype 3 or $\mathrm{P}_{2}$ also was predominant $(25,27)$. Pathotype 3 was shown to be highly virulent on all canola cultivars tested from Canada (27), and is also highly virulent on the spring oilseed rape differential host Brutor (Table 2). Therefore, this pathotype appears to be a reasonable choice to use in screening for clubroot resistance in B. napus canola in Alberta. However, caution should be used in any breeding strategy, because rare pathotypes of $P$. brassicae may quickly become predominant if susceptible host genotypes are continuously grown (20). Genetic resistance will have to be utilized in conjunction with other management strategies, including crop rotation and proper sanitation, to ensure its durability.

\section{ACKNOWLEDGMENTS}

We thank the Agriculture \& Food Council, the Alberta Crop Industry Development Fund, the Alberta Canola Producers Commission, and the Saskatchewan Canola Development Commission for financial support; S. Brezden and V. P. Manolii for technical assistance; R. C. Yang, Z. Wang, and Y. Yang for helpful discussions; and M. Harding for critically reviewing this manuscript.

\section{LITERATURE CITED}

1. Ayers, G. W. 1957. Races of Plasmodiophora brassicae. Can. J. Bot. 35:923-932.

2. Buczacki, S. T. 1977. Root infections from single resting spores of Plasmodiophora brassicae. Trans. Br. Mycol. Soc. 69:328-329.

3. Buczacki, S. T., Toxopeus, H., Mattusch, P., Johnston, T. D., Dixon, G. R., and Hobolth, L.
A. 1975. Study of physiologic specialization in Plasmodiophora brassicae: proposals for attempted rationalization through an international approach. Trans. Br. Mycol. Soc. 65:295-303.

4. Crute, I. R., Gray, A. R., Crisp, P., and Buczacki, S. T. 1980. Variation in Plasmodiophora brassicae and resistance to clubroot disease in Brassicas and allied crops-a critical review. Plant Breed. Abstr. 50:91-104.

5. Hildebrand, P. D., and Delbridge, R. W. 1995. Race survey of Plasmodiophora brassicae in Nova Scotia. Can. Plant Dis. Surv. 75:170.

6. Hildebrand, P. D., and McRae, K. D. 1998. Control of clubroot caused by Plasmodiophora brassicae with nonionic surfactants. Can. J. Plant Pathol. 20:1-11.

7. Honig, F. 1931. Der Kohlkropferreger (Plasmodiophora brassicae Wor.). Gartenbauwissenschaft 5:116-225.

8. Horiuchi, S., and Hori, M. 1980. A simple greenhouse technique for obtaining high levels of clubroot incidence. Bull. Chugoku Natl. Agric. Exp. Stn. Ser. E. (Environ. Div.) 17:3355 .

9. Jones D. R., Ingram, D. S , and Dixon, G. R. 1982. Characterization of isolates derived from single resting spores of Plasmodiophora brassicae and studies of their interaction. Plant Pathol. 31:239-246.

10. Kageyama, K., Kamimura, Y., and Hyakumachi, M. 1995. A simple inoculation method with a single resting spore of Plasmodiophora brassicae. Ann. Phytopathol. Soc. Jpn. 61:415418.

11. Karling, J. S. 1968. The Plasmodiophorales. Hafner Publishing Company, Inc., New York.

12. Klasse, H. J. 1996. Calcium cyanamide-an effective tool to control clubroot-a review. Acta Hortic. 407:403-409.

13. Kuginuki, Y., Yoshikawa, H.., and Hirai, M. 1999. Variation in virulence of Plasmodiophora brassicae in Japan tested with clubrootresistant cultivars of Chinese cabbage (Brassica rapa L. spp. pekinensis). Eur. J. Plant Pathol. 105:327-332.

14. Manzanares-Dauleux, M. J., Divaret, I., Baron, F., and Thomas, G. 2001. Assessment of biological and molecular variability between and within field isolates of Plasmodiophora brassicae. Plant Pathol. 50:165-173.

15. Naiki, T., and Dixon, G. R. 1987. The effects of chemicals on developmental stages of Plasmodiophora brassicae (clubroot). Plant Pathol. 36:316-327.

16. Pageau, D., Lajeunesse, J., and Lafond, J. 2006. Impact de l'hernie des crucifères [Plasmodiophora brassicae] sur la productivité du canola. Can. J. Plant Pathol. 28:137-143.

17. Reyes, A. A., Davidson, R. T., and Marks, C. F. 1974. Races, pathogenicity and chemical control of Plasmodiophora brassicae in Ontario. Phytopathology 64:173-177.

18. Rimmer, S. R., Kutcher, H. R., and Morrall, R. A. A. 2003. Diseases of canola and mustard. Pages129-146 in: Diseases of Field Crops in Canada. K. L. Bailey, B. D. Gossen, R. K.
Gugel, and R. A. A. Morrall, eds. Can. Phytopathol. Soc. Saskatoon, SK, Canada.

19. Scott, E. S. 1985. Production and characterization of single-spore isolates of Plasmodiophora brassicae. Plant Pathol. 34:287-292.

20. Seaman, W. L., Walker, J. C., and Larson, R. H. 1963. A new race of Plasmodiophora brassicae affecting Badger Shipper cabbage. Phytopathology 53:1426-1429.

21. Shah, D. A., and Madden, L. V. 2004. Nonparametric analysis of ordinal data in designed factorial experiments. Phytopathology 94:3343

22. Somé, A, Manzanares, M. J., Laurens, F., Baron, F., Thomas, G., and Rouxel, F. 1996. Variation for virulence on Brassica napus L. amongst Plasmodiophora brassicae collecions from France and derived single-spore isolates. Plant Pathol. 45:432-439.

23. Strelkov, S. E., Cao, T., Manolii V. P., Lange R. M., Smith-Degenhardt, E., Orchard, D., and Tewari, J. P. 2006. Incidence of clubroot on canola in Alberta in 2005. Can. Plant Dis. Surv. 86:91-93

24. Strelkov, S. E., Manolii, V. P., Cao, T., Hwang, S. F., and Orchard, D. 2007. Incidence of clubroot on canola in Alberta in 2006. Can. Plant Dis. Surv. 87:109-111.

25. Strelkov, S. E., Manolii, V. P., Cao, T., Xue, S. and Hwang, S. F. 2007. Pathotype classification of Plasmodiophora brassicae and its occurrence in Brassica napus in Alberta, Canada. J. Phytopathol. 155:706-712.

26. Strelkov, S. E., Tewari, J. P., Hartman, M., and Orchard, D. 2005. Clubroot on canola in Alberta in 2003 and 2004. Can. Plant Dis. Surv. 85:72-73.

27. Strelkov, S. E., Tewari, J. P., and SmithDegenhardt, E. 2006. Characterization of Plasmodiophora brassicae populations form Alberta Canada. Can. J. Plant Pathol. 28:467-474.

28. Suzuki, K., Matsumiya, E., Ueno, Y., and Mizutani, J. 1992. Some properties of germination-stimulating factor from plants for resting spores of Plasmodiophora brassicae. Ann. Phytopathol. Soc. Jpn. 58:699-705.

29. Tewari, J. P., Strelkov, S. E., Orchard, D., Hartman, M., Lange, R. M., and Turkington, T. K. 2005. Identification of clubroot of crucifers on canola (Brassica napus) in Alberta. Can. J. Plant Pathol. 27:143-144.

30. Tinggal, S. H., and Webster, J. 1981. Technique for single spore infection by Plasmodio phora brassicae. Trans. Br. Mycol. Soc. $87: 179-190$

31. Toxopeus, H., Dixon, G. R., and Mattusch, P. 1986. Physiological specialization in Plas modiophora brassicae: an analysis by international experimentation. Trans. Br. Mycol. Soc. 87:279-286.

32. Voorips, R. E. 1995. Plasmodiophora brassicae: aspects of pathogenesis and resistance in Brassica oleracea. Euphytica 83:139-146.

33. Williams, P. H. 1966. A system for the determination of races of Plasmodiophora brassicae that infect cabbage and rutabaga. Phytopathology 56:624-626. 